\title{
REVIEWS
}

\section{Salivary Gland Branching Morphogenesis - Recent Progress and Future Opportunities}

\author{
Jeff Chi-feng Hsu, Kenneth M. Yamada* \\ Laboratory of Cell and Developmental Biology, National Institute of Dental and Craniofacial Research, National Institutes of \\ Health, Bethesda, USA
}

\begin{abstract}
Salivary glands provide saliva to maintain oral health, and a loss of salivary gland function substantially decreases quality-of-life. Understanding the biological mechanisms that generate salivary glands during embryonic development may identify novel ways to regenerate function or design artificial salivary glands. This review article summarizes current research on the process of branching
\end{abstract}

morphogenesis of salivary glands, which creates gland structure during development. We highlight exciting new advances and opportunities in studies of cell-cell interactions, mechanical forces, growth factors, and gene expression patterns to improve our understanding of this important process.

Keywords salivary gland, epithelium, branching morphogenesis, fibronectin, E-cadherin, growth factor

Received Apr. 23, 2010; Revision accepted Jul. 7, 2010

\section{Introduction}

Salivary glands fulfill important roles in oral biology by producing saliva to provide water for lubrication, as well as supplying electrolytes, mucus, antibacterial compounds, and various enzymes to the oral cavity. Loss of normal salivary gland function can result in widespread deterioration of oral health. For example, salivary hypofunction and xerostomia caused by Sjögren's disease, irradiation for oral cancer, or side effects of medication can lead to dental caries, severe periodontal disease, persistent infections, and difficulties in mastication and swallowing. These effects can seriously affect the quality-of-life of patients.

Human salivary glands are generated in embryonic development by the process of branching morphogenesis. Understanding the biological principles of this process may not only advance our understanding of developmental biology, but it could also provide novel approaches to tissue engineering for regenerating salivary glands or for creating an artificial salivary gland or other organs to restore missing function.

Branching morphogenesis is a key developmental process commonly utilized to generate the functionally efficient, complex, but well-ordered, tissue architecture found in many organs, including lung, kidney, mammary and salivary gland (Hogan, 1999). Although different regulatory mechanisms are employed temporally and spatially at specific developmental stages of each organ, there also appear to be common mechanisms shared by multiple branched organs (Davies, 2002; Lu and Werb, 2008). Hence, understanding the mechanism of morphogenesis of one type of branched organ can potentially provide significant insight to that of another.

The mouse salivary gland, specifically the submandibular gland, has been used frequently as a 
model for the general process of branching morphogenesis because of its clearly defined branching pattern, the relative ease of gland isolation, and the availability of a well-established system for ex vivo gland culture. Detailed protocols for ex vivo culture of submandibular glands (SMG) have been described recently (Sakai and Onodera, 2008; Rebustini and Hoffman, 2009). The gland starts as a simple spherical epithelial structure attached to a single epithelial stalk and is surrounded by condensed mesenchyme at embryonic day 12 (E12). Branching is initiated at E13 by the formation of shallow clefts that deepen and subdivide the epithelium into multiple buds. The gland then undergoes repetitive rounds of cleft and bud formation, as well as bud/duct elongation, to form a highly branched structure by E14 to E15 (Figure 1). Branching continues over the subsequent days of embryonic development. During this process, thousands (or more) intricate and dynamic interactions occur within, as well as between, the epithelium and the mesenchyme (the two main cell populations found in the early gland). These interactions are affected by factors that include, but are not limited to, changes in extracellular matrix protein synthesis and distribution, alterations in cell-cell and cellmatrix adhesions, and changes in cell growth and contractility. How these interactions help to sculpt the form of the gland has been a central focus in understanding branching morphogenesis. In this review, we examine current research topics and studies emphasizing the past five years investigating the mechanisms and regulation of mouse salivary gland branching morphogenesis. We highlight exciting new opportunities to improve our understanding of this important process.

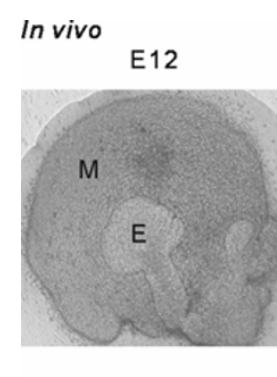

Ex vivo culture

$2 \mathrm{~h}$

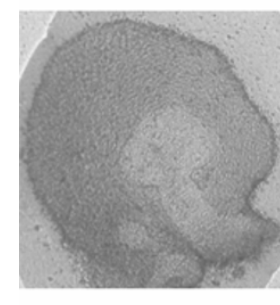

E13

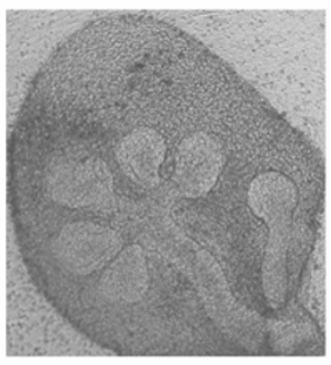

$24 \mathrm{~h}$

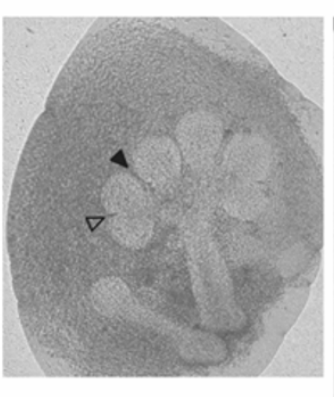

E14

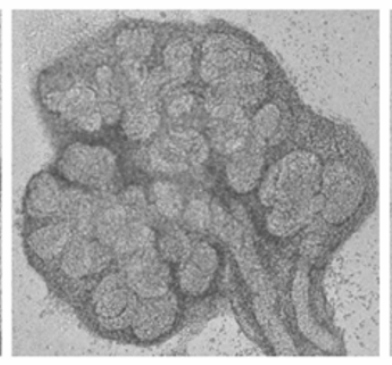

$48 \mathrm{~h}$

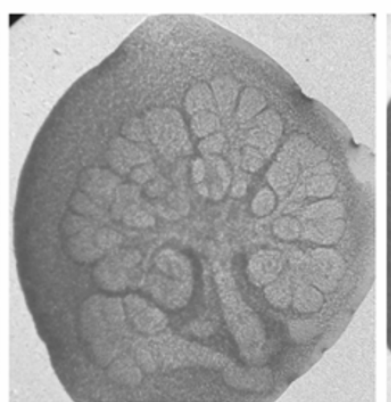

E15

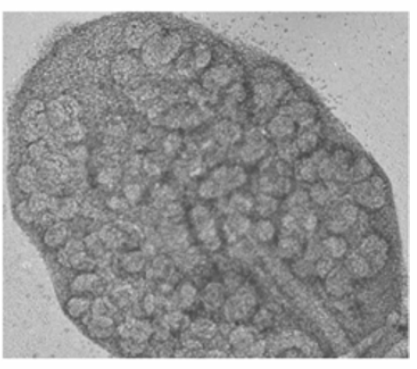

$72 \mathrm{~h}$

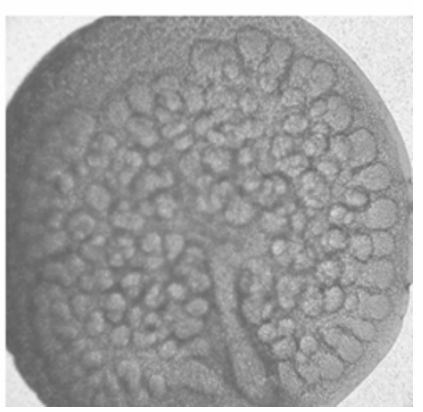

Figure 1 Branching morphogenesis of SMG in vivo and in ex vivo culture

Top panel: Mouse SMG isolated from embryonic day 12 (E12) through E15 illustrate the formation of a highly branched structure from a single epithelial bud. Bottom panel: The pattern of in vivo branching morphogenesis can be closely reproduced by ex vivo culture of an E12 SMG. $M$ denotes mesenchyme, $E$ denotes epithelium, and empty and filled arrowheads show examples of early and deepening clefts, respectively.

\section{Mechanical forces generated by the extracellular matrix}

The formation and deepening of epithelial clefts are critical initial events during SMG branching morphogenesis. These processes help to generate the multi-lobed structure of the gland by subdividing a simple epithelial bud into multiple smaller buds that elongate by forming secondary ducts and expand in size distally. From the first 
studies of the mechanisms of salivary branching morphogenesis, it has been clear that extracellular matrix (ECM) molecules play an important role in gland development. They are essential elements of tissue and organ architecture throughout the body and could potentially provide structural guidance cues to help "mold" the gland into its desired form. One model proposes that clefts are formed as a result of contraction or pulling of bundles of ECM fibrils by mesenchymal cells to generate mechanical forces that push inward at local sites against the growing epithelial bud at the epithelialmesenchymal interface. In support of this proposal, fibrillar structures were observed aligned along the base of clefts (Nakanishi et al., 1986). More recently, mathematical modeling has shown that it is possible to generate epithelial clefts that morphologically resemble naturally occurring clefts when appropriate mesenchymal forces are applied to an epithelial bud (Wan et al., 2008). It was suggested initially that the force-exerting fibers are comprised of the ECM protein collagen, since several types of collagen accumulate at the epithelial cleft sites (Nakanishi et al., 1988; Hayakawa et al., 1992), and chemical inhibition of collagen synthesis or addition of collagenase dramatically blocks branching of SMG (Spooner and Faubion, 1980; Fukuda et al., 1988; Nakanishi et al., 1988; Hayakawa et al., 1992). Although these findings highlight the importance of collagen accumulation and turnover during SMG branching, whether collagen accumulation in the clefts is involved in actively generating clefts or in merely stabilizing clefts by providing resistance to epithelial forces is currently not yet clear.

In fact, many researchers are now discovering new functions for a variety of ECM proteins. Additional roles for collagen have been suggested in a recent study reporting the discovery that the collagenolytic membrane-type matrix metalloproteinase MT-MMP2 releases bioactive $\mathrm{NC} 1$ domains from collagen IV in the basement membrane to drive epithelial proliferation (Rebustini et al., 2009). Moreover, the ECM proteins laminin and perlecan, which are both components of the basement membrane that lines the junction between epithelium and mesenchyme, have also been found to accumulate in early clefts. Examination of glands isolated from laminin $\alpha 5^{-/}$mice as well as
siRNA knockdown of laminin $\alpha 5$ demonstrate a reciprocal regulatory pathway between laminin $\alpha 5$ and fibroblast growth factor (FGF) signaling (Rebustini et al., 2007).

In addition, proteoglycans can play regulatory roles. Cleavage of heparan sulfate moieties from the basement membrane proteoglycan perlecan releases FGF10, which then acts on the epithelium to induce increased branching (Patel et al., 2007). To add another level of complexity, the size and sulfation patterns of heparan sulfate exert differential levels of modulation of the affinity of FGF10 binding and hence on the effect of FGF10 on epithelial branching morphogenesis (Patel et al., 2008).

The ECM protein fibronectin has also been identified as a putative cleft initiator (Sakai et al., 2003). It accumulates in the clefts and appears to direct its own inward translocation deeper into the cleft as cleft progression occurs (Larsen et al., 2006). Knockdown of fibronectin by siRNA prevents cleft formation in E12 SMGs, while the addition of exogenous fibronectin accelerates epithelial cleft formation while producing decreased E-cadherin-mediated cell-cell adhesions. The key regulator which provides a mechanistic link between fibronectin and cleft propagation has recently been identified as BTB (POZ) domain containing 7 (Btbd7) (Onodera et al., 2010). Interestingly, inhibiting cellular contractility by blocking Rhoassociated coiled-coil containing kinase (ROCK) I or non-muscle myosin II prevents cleft progression and freezes clefts in an early, unstable state (Daley et al., 2009). The authors suggest that at this stage, there is a "mechanochemical" checkpoint during which transition of clefts to a stabilized form is achieved through ROCKdependent assembly of fibronectin and potentially fibronectin-induced epithelial proliferation (Figure 2). An important future direction will be to determine mechanistically how fibronectin actually stimulates cleft formation and its progression, which could occur either directly or indirectly through intermediate mediator(s) that modulate cell-cell adhesive interactions locally and/or regulate motility. Taken together, these recent findings suggest that even though mechanical forces clearly play an important role in modulating SMG branching, there exists a much more complex level 


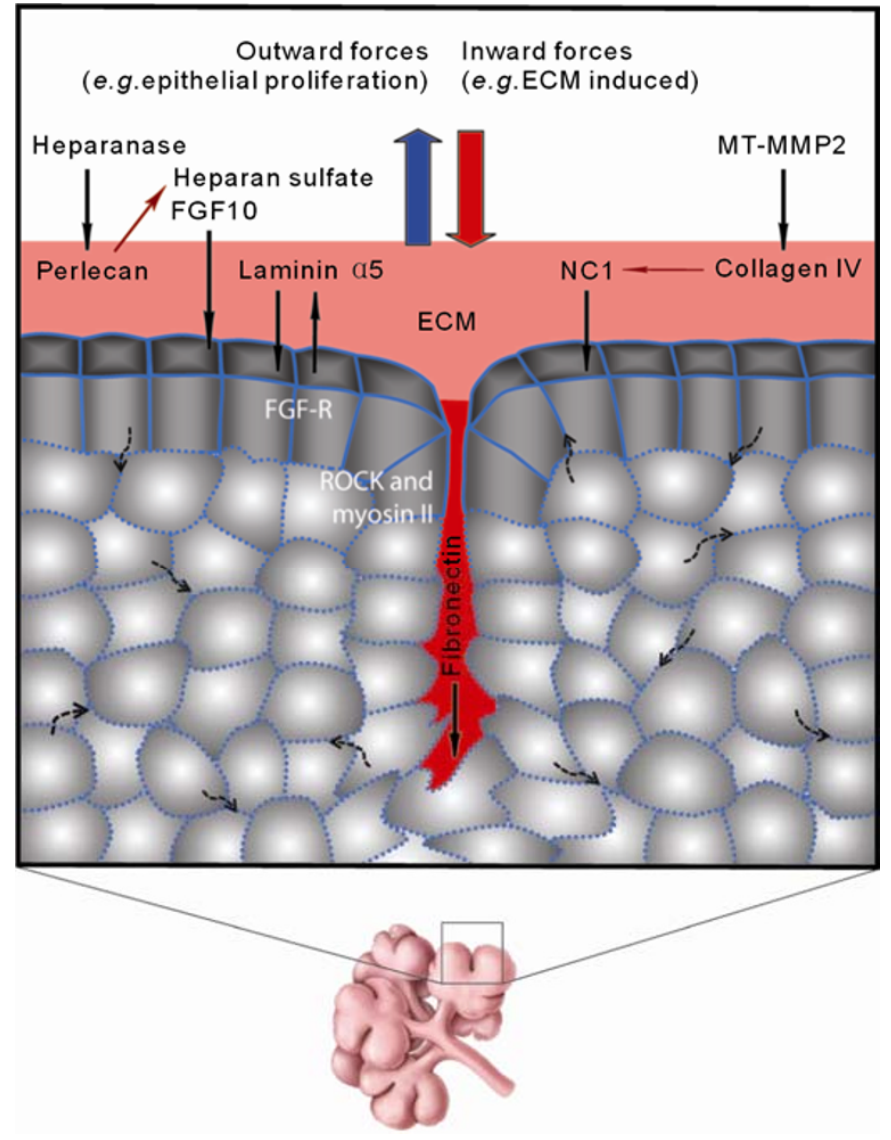

Figure 2 Morphogenetic changes

\begin{abstract}
Schematic diagram of dynamic changes in the morphology of a salivary epithelial bud as a result of multiple physical and signaling interactions. They include outward and inward forces generated by epithelial proliferation and ECM-mediated effects, respectively, regulation by growth factors, ECM, and enzymes that cleave ECM molecules. Boxed region shows part of an E13 SMG with a developing cleft. Red shading denotes ECM molecules. Dotted and solid blue lines denote E-cadherin. Solid black arrows denote direction of action. Solid thin red arrows denote generation of cleavage fragments. Dotted black arrows denote the dynamic and rapid motility of cells within the epithelium. Note that the mesenchymal cells are not shown here, but they surround the salivary epithelium in vivo.
\end{abstract}

of regulation of this process. It will be useful to delve deeper into the complex interplay between the mechanical forces exerted by the ECM, the cells that deposit the ECM, as well as the signaling pathways that induce these cells or are induced by the ECM.

\section{Cell-cell interactions}

The dramatic changes in structure and cellular organization that occur during SMG branching morphogenesis could be guided not only by mechanical forces, but also by dynamic interactions between neighboring cells. In fact, increasing evidence points to a critical role for the major cell-cell adhesion protein known as epithelial (E)-cadherin during SMG development. When E-cadherin is bound by its $\mathrm{C}$-terminus to various proteins including p120-catenin, $\beta$-catenin, and their associated proteins, it forms homotypic interactions with other E-cadherin molecules on a neighboring cell and mediates adhesive cell-cell interactions (Gumbiner, 2005). The importance of E-cadherin in SMG branching morphogenesis was established recently by antibody, RNA interference, and gene ablation approaches. SMG branching is inhibited in ex vivo cultures of SMG treated with E-cadherin-specific siRNA or function-blocking anti-E-cadherin antibodies (Walker et al., 2008). SMG epithelial cells dissociated enzymatically into single cells retain the potential to self-organize; they form cellular 
aggregates that proceed to undergo branching morphogenesis to form tissues with structural features and differentiation markers characteristic of the intact gland (Wei et al., 2007). E-cadherin plays an important role in this self-organizing process, since it is inhibited by function-blocking anti-E-cadherin antibodies.

E-cadherin is also important for SMG acinar differentiation, since SMG from postnatal p120catenin knock-out mice (with E-cadherin defects) had no acini and were comprised entirely of ducts (Davis and Reynolds, 2006). When p120-catenin was ablated between E13.5 and E14.5 (with associated loss of E-cadherin), abnormal phenotypes appeared, including severe mis-localization of polarity markers, aberrant or premature lumen dilation, cell shedding, and ductal epithelial protrusion/outgrowth into the lumen of ducts. Although the exact role of E-cadherin in SMG development is currently still unclear at a mechanistic level, these findings suggest that E-cadherin is critically needed to regulate or mediate SMG epithelial self-organization, branching, and acinus formation. In addition, the observation by Davis and Reynolds of abnormal luminal epithelial outgrowth (Davis and Reynolds, 2006) suggests that E-cadherin is involved in maintaining the integrity of SMG epithelial surfaces. This possibility is particularly interesting, since previous findings have demonstrated that even though epithelial E-cadherin expression is constitutive throughout all stages of SMG development, other key cell-cell junctional proteins are transiently lost. Low or even absence of expression of other junctional proteins such as desmoplakins I/II (major desmosomal proteins) and ZO-1 (a tight junction protein) occurs in early developing (E12 through to E14) SMG epithelia (Hieda et al., 1996). The well-known importance of these latter junctional proteins in securing stable cell-cell adhesions, as well as their strong lumenal up-regulated expression at later stages (E14 onwards), points to the possibility of differential temporal and region-specific regulation of cell-cell interactions during SMG epithelial morphogenesis. Consistent with the possibility that E-cadherin functions in place of other junctional proteins to stabilize external epithelial surfaces, the localization of E-cadherin is considerably stronger between cell junctions of outer bud epithelial cells than those between the inner bud cells (Walker et al., 2008). However, when a cleft forms to interrupt this layer of outer epithelial cells, E-cadherin localization and expression diminish as cell shift from cell-cell to cell-matrix adhesion (Sakai et al., 2003).

Interestingly, even though E-cadherin is expressed throughout SMG epithelia, many individual epithelial cells within epithelial bud(s) of earlystage SMG migrate very rapidly in a relatively random fashion (Larsen et al., 2006). This capacity for cell movement - with swapping of cell partners within a relative short period of time - implies either a weakened cell-cell adhesive function of the E-cadherin molecules present at significant levels or the existence of a dynamic adhesion "switch" that rapidly associates and dissociates to allow rapid cell motility; either mechanism could give the gland its plasticity to allow the rapid formation of clefts (Figure 2). With the very recent establishment of the p120-catenin-E-cadherin complex crystal structure (Ishiyama et al., 2010), it may not be long before we understand how changes in adhesion protein complexes involving E-cadherin and other molecules can regulate this pattern of cell motility, especially at local sites such as in the cleft region of the SMG.

\section{Morphogenetic roles of growth factors}

Growth factors are well-established regulators of critical functions throughout all phases of embryonic development in both vertebrates and invertebrates. The ability of growth factors to stimulate cell proliferation and to modulate cell motility and migration through complex arrays of interconnected signaling pathways has been extensively characterized and studied in two-dimensional cell culture systems (Alberts et al., 2002). There is increasing knowledge about potentially important signaling pathways in salivary gland development, which are starting to be unified using systems biology approaches (Melnick et al., 2009; Larsen et al., 2010). A major opportunity for the future will be to apply computational modeling to systems biology analyses of specific stages of salivary gland development to provide a more 
unified, holistic understanding of the complex signaling necessary for efficient gland formation at each phase of development.

The best-studied growth factor/receptor pairs essential for SMG development are the fibroblast growth factor (FGF)/receptor and the epidermal growth factor (EGF)/receptor. In ex vivo cultured SMG, branching morphogenesis involves both FGFR signaling (Morita and Nogawa, 1999; Hoffman et al., 2002; Jaskoll et al., 2002) and EGFR signaling (Kashimata and Gresik, 1997; Kashimata et al., 2000; Umeda et al., 2001). Transgenic mice lacking FGF8 (Daphna-Iken et al., 1998), FGF10 (Ohuchi et al., 2000), FGFR2b (De Moerlooze et al., 2000), or EGFR (Jaskoll and Melnick, 1999) do not develop past the initial bud stage ( $F g f 8$-conditional null mice, Fgfl0-null, and $F G F R 2 b$-null mice), or they have substantially fewer terminal buds (EGFR-null mice). These studies indicate that each of these two growth factor-receptor systems is necessary for at least one essential, nonredundant function during normal SMG development.

When supplemented with appropriate growth factor(s), mesenchyme-free epithelial rudiments from mouse embryos undergo branching when cultured ex vivo, though the process eventually stops. This modified version of ex-vivo SMG culture simplifies the system by eliminating potential contributions from the mesenchyme in regulating branching morphogenesis. Therefore, this system is commonly used as a model to study the roles of specific growth factors on epithelial morphogenesis. Using this model, EGF was shown to stimulate bud formation (Morita and Nogawa, 1999), while FGF7 and FGF10 differentially promote epithelial bud formation and bud/duct elongation, respectively (Steinberg et al., 2005). A recent study has attempted to dissect the signaling components that contribute to the epithelial phenotypes induced by EGF, FGF7, and FGF10 (Koyama et al., 2008). Previous studies had shown that a number of signaling pathways were important for SMG branching morphogenesis, including mitogen-activated protein kinases (ERK-1/2), phospholipase $\mathrm{C} \gamma 1$ (PLC $\gamma 1$ ), and phosphatidylinositol-3-kinase (PI3K) (Kashimata et al., 2000; Koyama et al., 2003; Larsen et al., 2003; Steinberg et al., 2005). Comparisons of the ability of these three growth factors to stimulate each of these pathways, and importantly the ability of specific inhibitors to block their induced epithelial phenotypes, suggest that ERK-1/2 (stimulated by EGF and FGF7) is important for bud formation, whereas PLC $\gamma 1$ (stimulated by FGF7 and FGF10) may be important for bud/duct elongation. The fact that there are multiple common signaling pathways employed by these three different growth factors implies that during SMG development, there needs to be cross-regulation between different growth factor receptor systems. In fact, this coordination was demonstrated in two recent studies. E12 SMG epithelial rudiments do not branch when cultured with EGF and lysophosphatidic acid unless they are pre- or co-cultured with FGF7 or FGF10 (Nitta et al., 2009). The authors suggest that priming by FGF of the EGFR system is important for EGFR-mediated induction of branching morphogenesis. In another study, a potential upstream regulator of FGFR signaling may have been identified by the demonstration that platelet-derived growth factor (PDGF) receptor signaling is important for SMG branching morphogenesis; the authors suggest that the stimulatory effect is due to its ability to up-regulate FGF expression (Yamamoto et al., 2008). The results of these recent findings are summarized in Figure 3. It will be important to continue to explore how these and other different growth factor receptormediated signal transduction pathways communicate with each other. It will also be important to determine how these signaling pathways regulate, or are regulated by, the ECM and by cell-cell interactions, and how all these regulatory interactions are integrated to form the functional gland.

\section{Other future opportunities}

\section{Genomics}

A new public database is now available through the Intramural Program of the National Institute of Dental and Craniofacial Research to accelerate salivary gland research. The website for this Salivary Molecular Anatomy Project (SGMAP) is accessible at http://sgmap.nidcr.nih.gov/ to obtain 


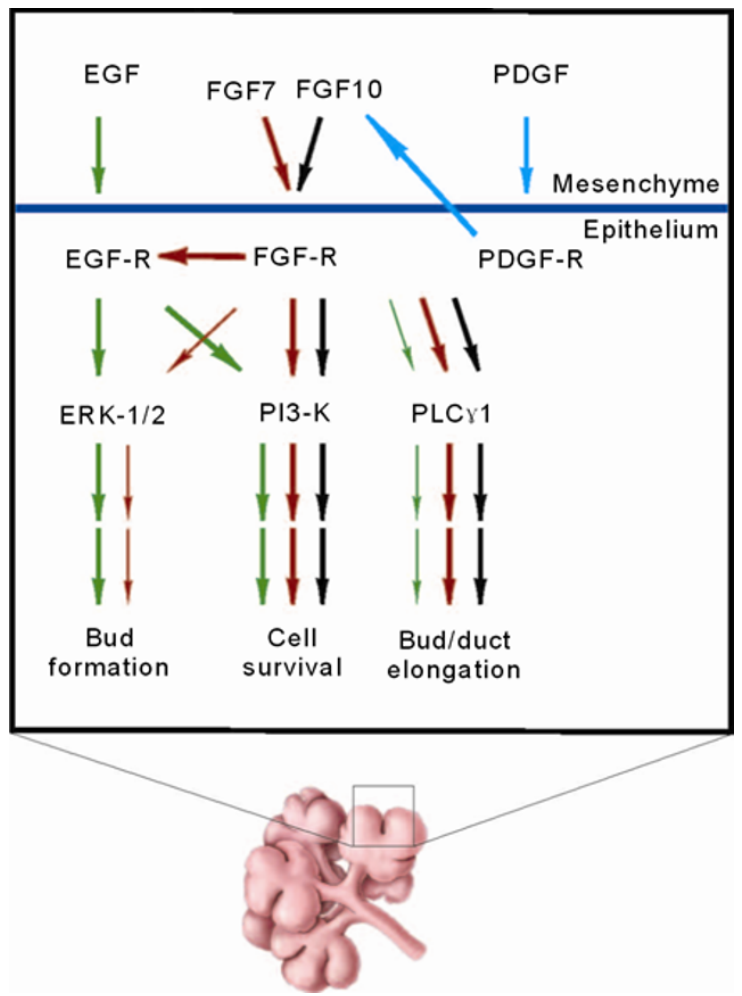

Figure 3 Signaling

Schematic diagram of growth factor and signal transduction pathways implicated in salivary gland branching morphogenesis. Arrows indicate positive stimulation.

gene expression data for a full range of developmental stages of mouse salivary glands, as well as site-specific expression patterns. The database can be searched readily by gene symbol (e.g., fgf), gene description (e.g., zinc finger), or gene ontology term (e.g., transcription factor). Specific patterns of spatial or temporal expression can also be searched from within the site to find other genes expressed in patterns similar to an initially identified gene. This tool should provide novel approaches to analyzing gland development and sources of new hypotheses for future projects.

\section{Tissue engineering}

Basic research knowledge about the mechanisms of salivary gland development should facilitate future programs to regenerate or produce an artificial salivary gland to alleviate xerostomia. Encouraging progress has been made to develop the technologies needed for an artificial gland (Baum and Tran, 2006; Aframian et al., 2007). Once the mechanisms of branching morphogenesis are understood, it may be possible to dedifferentiate adult human salivary gland cells to the cellular developmental stage appropriate for selfassembly into functional gland units. For example, suspensions of human epithelial and mesenchymal cells dedifferentiated to mimic the cells of embryonic salivary glands might directly self-organize and initiate differentiation, as shown for cells from developing mouse salivary glands (Wei et al., 2007). This type of approach would probably need to be combined with biomaterials approaches to organize the gland into a functional secretory system with a duct leading to the oral cavity. Interestingly, the biomaterial chitosan can promote branching morphogenesis (Yang and Young, 2009), suggesting that biomaterials could provide both physical frameworks and stimuli for constructing an artificial salivary gland. In addition, however, existing salivary glands could also be used as a source of secreted bioactive proteins such as insulin by the use of viral gene transfer methods (Aframian et al., 2007).

An alternative or complementary approach to 
salivary gland tissue engineering will be the use of stem or progenitor cells transplantation into damaged salivary glands. If stem cells can be isolated from salivary glands of patients, for example prior to administration of radiation therapy to treat head and neck carcinoma, it may be possible to transplant these cells back into patients to restore salivary gland function. The feasibility of this approach has already been demonstrated in a mouse model system (Lombaert et al., 2008). Ongoing investigations to identify reliable markers for salivary gland stem/progenitor cells and to use growth factors to guide cell fate decisions for effective differentiation should accelerate research in this promising area (Lombaert and Hoffman, 2010). Ultimately, a combined approach involving the use of an artificial gland framework/scaffold with appropriately transplanted stem/progenitor cells with the addition of selected growth factors may provide a solution to restoring salivary function in patients with defective salivary glands.

Taken together, future progress in research on salivary gland development and salivary tissue engineering will require the combined efforts of many different types of researchers from fields as diverse as cell biology, developmental biology, biomaterials research, systems biology, clinical research, and other types of expertise. Public databases and multidisciplinary teams combining varying types of scientific expertise will accelerate research. There are many exciting opportunities for the future.

\section{Acknowledgment}

Supported by the Intramural Research Program of the National Institute of Dental and Craniofacial Research, NIH.

\section{References}

Aframian DJ, Amit D, David R, Shai E, Deutsch D, Honigman A, et al. (2007). Reengineering salivary gland cells to enhance protein secretion for use in developing artificial salivary gland device. Tissue Eng, 13(5): 995-1001.

Alberts B, Johnson A, Lewis J, Raff M, Roberts K, Walter P (2002). Molecular Biology of the Cell. 4th ed. New
York: Garland Science.

Baum BJ, Tran SD (2006). Synergy between genetic and tissue engineering: creating an artificial salivary gland. Periodontol 2000, 41(1): 218-223.

Daley WP, Gulfo KM, Sequeira SJ, Larsen M (2009). Identification of a mechanochemical checkpoint and negative feedback loop regulating branching morphogenesis. Dev Biol, 336(2): 169-182.

Daphna-Iken D, Shankar DB, Lawshe A, Ornitz DM, Shackleford GM, MacArthur CA (1998). MMTV-Fgf8 transgenic mice develop mammary and salivary gland neoplasia and ovarian stromal hyperplasia. Oncogene, 17(21): 2711-2717.

Davies JA (2002). Do different branching epithelia use a conserved developmental mechanism? Bioessays, 24(10): 937-948.

Davis MA, Reynolds AB (2006). Blocked acinar development, E-cadherin reduction, and intraepithelial neoplasia upon ablation of p120-catenin in the mouse salivary gland. Dev Cell, 10(1): 21-31.

De Moerlooze L, Spencer-Dene B, Revest JM, Hajihosseini M, Rosewell I, Dickson C (2000). An important role for the IIIb isoform of fibroblast growth factor receptor 2 (FGFR2) in mesenchymal-epithelial signalling during mouse organogenesis. Development, 127(3): 483-492.

Fukuda Y, Masuda Y, Kishi J, Hashimoto Y, Hayakawa T, Nogawa H, et al. (1988). The role of interstitial collagens in cleft formation of mouse embryonic submandibular gland during initial branching. Development, 103(2): 259-267.

Gumbiner BM (2005). Regulation of cadherin-mediated adhesion in morphogenesis. Nat Rev Mol Cell Biol, 6(8): 622-634.

Hayakawa T, Kishi J, Nakanishi Y (1992). Salivary gland morphogenesis: possible involvement of collagenase. Matrix, 1(Suppl): 344-351.

Hieda Y, Iwai K, Morita T, Nakanishi Y (1996). Mouse embryonic submandibular gland epithelium loses its tissue integrity during early branching morphogenesis. Dev Dyn, 207(4): 395-403.

Hoffman MP, Kidder BL, Steinberg ZL, Lakhani S, Ho S, Kleinman HK, et al. (2002). Gene expression profiles of mouse submandibular gland development: FGFR1 regulates branching morphogenesis in vitro through BMP- and FGF-dependent mechanisms. Development, 129(24): 5767-5778.

Hogan BL (1999). Morphogenesis. Cell, 96(2): 225-233.

Ishiyama N, Lee SH, Liu S, Li GY, Smith MJ, Reichardt 
LF, et al. (2010). Dynamic and static interactions between p120 catenin and E-cadherin regulate the stability of cell-cell adhesion. Cell, 141(1): 117-128.

Jaskoll T, Melnick M (1999). Submandibular gland morphogenesis: stage-specific expression of TGF-alpha/ EGF, IGF, TGF-beta, TNF, and IL-6 signal transduction in normal embryonic mice and the phenotypic effects of TGF-beta2, TGF-beta3, and EGF-r null mutations. Anat Rec, 256(3): 252-268.

Jaskoll T, Zhou YM, Chai Y, Makarenkova HP, Collinson JM, West JD, et al. (2002). Embryonic submandibular gland morphogenesis: stage-specific protein localization of FGFs, BMPs, Pax6 and Pax9 in normal mice and abnormal SMG phenotypes in FgfR2-IIIc(+/Delta), BMP7(-/-) and Pax6(-/-) mice. Cells Tissues Organs, 170(2/3): 83-98.

Kashimata M, Gresik EW (1997). Epidermal growth factor system is a physiological regulator of development of the mouse fetal submandibular gland and regulates expression of the alpha6-integrin subunit. Dev Dyn, 208(2): 149-161.

Kashimata M, Sayeed S, Ka A, Onetti-Muda A, Sakagami H, Faraggiana T, et al. (2000). The ERK-1/2 signaling pathway is involved in the stimulation of branching morphogenesis of fetal mouse submandibular glands by EGF. Dev Biol, 220(2): 183-196.

Koyama N, Hayashi T, Ohno K, Siu L, Gresik EW, Kashimata M (2008). Signaling pathways activated by epidermal growth factor receptor or fibroblast growth factor receptor differentially regulate branching morphogenesis in fetal mouse submandibular glands. Dev Growth Differ, 50(7): 565-576.

Koyama N, Kashimata M, Sakashita H, Sakagami H, Gresik EW (2003). EGF-stimulated signaling by means of PI3K, PLCgamma1, and PKC isozymes regulates branching morphogenesis of the fetal mouse submandibular gland. Dev Dyn, 227(2): 216-226.

Larsen M, Hoffman MP, Sakai T, Neibaur JC, Mitchell JM, Yamada KM (2003). Role of PI 3-kinase and PIP3 in submandibular gland branching morphogenesis. Dev Biol, 255(1): 178-191.

Larsen M, Wei C, Yamada KM (2006). Cell and fibronectin dynamics during branching morphogenesis. J Cell Sci, 119(Pt 16): 3376-3384.

Larsen M, Yamada KM, Musselmann K (2010). Systems analysis of salivary gland development and disease. Wiley Interdisciplinary Reviews: Systems Biology and Medicine, http://wires.wiley.com/WileyCDA/WiresArticle /wisId-WSBM94.html.
Lombaert IM, Brunsting JF, Wierenga PK, Faber H, Stokman MA, Kok T, et al. (2008). Rescue of salivary gland function after stem cell transplantation in irradiated glands. PLoS One, 3(4): e2063.

Lombaert IM, Hoffman MP (2010). Epithelial stem/ progenitor cells in the embryonic mouse submandibular gland. Front Oral Biol, 14: 90-106.

Lu P, Werb Z (2008). Patterning mechanisms of branched organs. Science, 322(5907): 1506-1509.

Melnick M, Phair RD, Lapidot SA, Jaskoll T (2009). Salivary gland branching morphogenesis: a quantitative systems analysis of the Eda/Edar/NFkappaB paradigm. BMC Dev Biol, 9: 32.

Morita K, Nogawa H (1999). EGF-dependent lobule formation and FGF7-dependent stalk elongation in branching morphogenesis of mouse salivary epithelium in vitro. Dev Dyn, 215(2): 148-154.

Nakanishi Y, Nogawa H, Hashimoto Y, Kishi J, Hayakawa $\mathrm{T}$ (1988). Accumulation of collagen III at the cleft points of developing mouse submandibular epithelium. Development, 104(1): 51-59.

Nakanishi Y, Sugiura F, Kishi J, Hayakawa T (1986). Scanning electron microscopic observation of mouse embryonic submandibular glands during initial branching: preferential localization of fibrillar structures at the mesenchymal ridges participating in cleft formation. J Embryol Exp Morphol, 96(1): 65-77.

Nitta M, Kume T, Nogawa H (2009). FGF alters epithelial competence for EGF at the initiation of branching morphogenesis of mouse submandibular gland. Dev Dyn, 238(2): 315-323.

Ohuchi H, Hori Y, Yamasaki M, Harada H, Sekine K, Kato $\mathrm{S}$, et al. (2000). FGF10 acts as a major ligand for FGF receptor $2 \mathrm{IIIb}$ in mouse multi-organ development. Biochem Biophys Res Commun, 277(3): 643-649.

Onodera T, Sakai T, Hsu JC, Matsumoto K, Chiorini JA, Yamada KM (2010). Btbd7 regulates epithelial cell dynamics and branching morphogenesis. Science, 329 (5991): 562-565.

Patel VN, Knox SM, Likar KM, Lathrop CA, Hossain R, Eftekhari S, et al. (2007). Heparanase cleavage of perlecan heparan sulfate modulates FGF10 activity during ex vivo submandibular gland branching morphogenesis. Development, 134(23): 4177-4186.

Patel VN, Likar KM, Zisman-Rozen S, Cowherd SN, Lassiter KS, Sher I, et al. (2008). Specific heparan sulfate structures modulate FGF10-mediated submandibular gland epithelial morphogenesis and differentiation. J Biol Chem, 283(14): 9308-9317. 
Rebustini IT, Hoffman MP (2009). ECM and FGFdependent assay of embryonic SMG epithelial morphogenesis: investigating growth factor/matrix regulation of gene expression during submandibular gland development. Methods Mol Biol, 522: 319-330.

Rebustini IT, Myers C, Lassiter KS, Surmak A, Szabova L, Holmbeck K, et al. (2009). MT2-MMP-dependent release of collagen IV NC1 domains regulates submandibular gland branching morphogenesis. Dev Cell, 17(4): 482-493.

Rebustini IT, Patel VN, Stewart JS, Layvey A, GeorgesLabouesse E, Miner JH, et al. (2007). Laminin alpha5 is necessary for submandibular gland epithelial morphogenesis and influences FGFR expression through beta1 integrin signaling. Dev Biol, 308(1): 15-29.

Sakai T, Larsen M, Yamada KM (2003). Fibronectin requirement in branching morphogenesis. Nature, 423(6942): 876-881.

Sakai T, Onodera T (2008). Embryonic organ culture. Curr Protoc Cell Biol, Chapter 19: Unit 19.08.

Spooner BS, Faubion JM (1980). Collagen involvement in branching morphogenesis of embryonic lung and salivary gland. Dev Biol, 77(1): 84-102.

Steinberg Z, Myers C, Heim VM, Lathrop CA, Rebustini IT, Stewart JS, et al. (2005). FGFR2b signaling regulates ex vivo submandibular gland epithelial cell proliferation and branching morphogenesis. Development, 132(6): 1223-1234.
Umeda Y, Miyazaki Y, Shiinoki H, Higashiyama S, Nakanishi Y, Hieda Y (2001). Involvement of heparinbinding EGF-like growth factor and its processing by metalloproteinases in early epithelial morphogenesis of the submandibular gland. Dev Biol, 237(1): 202-211.

Walker JL, Menko AS, Khalil S, Rebustini I, Hoffman MP, Kreidberg JA, et al. (2008). Diverse roles of E-cadherin in the morphogenesis of the submandibular gland: insights into the formation of acinar and ductal structures. Dev Dyn, 237(11): 3128-3141.

Wan X, Li Z, Lubkin SR (2008). Mechanics of mesenchymal contribution to clefting force in branching morphogenesis. Biomech Model Mechanobiol, 7(5): 417-426.

Wei C, Larsen M, Hoffman MP, Yamada KM (2007). Self-organization and branching morphogenesis of primary salivary epithelial cells. Tissue Eng, 13(4): 721-735.

Yamamoto S, Fukumoto E, Yoshizaki K, Iwamoto T, Yamada A, Tanaka K, et al. (2008). Platelet-derived growth factor receptor regulates salivary gland morphogenesis via fibroblast growth factor expression. J Biol Chem, 283(34): 23139-23149.

Yang TL, Young TH (2009). The specificity of chitosan in promoting branching morphogenesis of progenitor salivary tissue. Biochem Biophys Res Commun, 381(4): 466-470.

*Corresponding author: Kenneth M. Yamada

Address: National Institute of Dental and Craniofacial Research, NIH - Laboratory of Cell and Developmental Biololgy,

Building 30, Room 426, NIH, 30 Convent Drive MSC 4370, Bethesda, Maryland 20892-4370, USA

Tel: $0013014969061 \quad$ E-mail: kenneth.yamada@nih.gov 